\title{
Fungal infections in adult patients on extracorporeal life support
}

\author{
Yiorgos Alexandros Cavayas ${ }^{1 *}$ (D), Hakeem Yusuff ${ }^{2}$ and Richard Porter $^{2}$
}

\begin{abstract}
Background: Patients on extracorporeal membrane oxygenation (ECMO) are often among the most severely ill in the intensive care unit. They are often receiving broad-spectrum antibiotics; they have multiple entry points for pathogens; and their immune system is impaired by blood circuit interaction. These factors are thought to predispose them to fungal infections. We thus aimed to evaluate the prevalence, risk factors, and prognosis of fungal infections in adults on ECMO.

Methods: We conducted a retrospective cohort study using the Extracorporeal Life Support Organization registry, which compiles data on ECMO use from hundreds of international centers. We included all adult patients from 2006 to 2016 on any mode of ECMO with either a diagnosis of fungal infection or a positive fungal culture.

Results: Our study comprised 2129 adult patients (10.8\%) with fungal colonization or infection. Aspergillus involvement (colonization or infection) was present in 272 patients (1.4\%), of whom 35.7\% survived to hospital discharge. There were 245 patients (1.2\%) with Candida invasive bloodstream infection, with 35.9\% survival. Risk factors for Aspergillus involvement included solid organ transplant (OR 1.83; $p=0.008$ ), respiratory support (OR 2.75; $p<0.001)$, and influenza infection (OR 2.48; $p<0.001)$. Risk factors for candidemia included sepsis (OR 1.60; $p=0$. $005)$ and renal replacement therapy (OR 1.55; $p=0.007$ ). In multivariable analysis, Aspergillus involvement (OR 0.40; $p<0.001)$ and candidemia (OR 0.47; $p<0.001$ ) were both independently associated with decreased survival.
\end{abstract}

Conclusions: The prevalence of Aspergillus involvement and Candida invasive bloodstream infection were not higher in patients on ECMO than what has been reported in the general intensive care population. Both were independently associated with a reduced survival. Aspergillus involvement was strongly associated with ECMO for respiratory support and influenza.

Keywords: Extracorporeal membrane oxygenation [D015199], Invasive fungal infections [D000072742], Aspergillus [D001230], Aspergillosis [D001228], Candida [D002175], Candidemia [D058387]

\section{Background}

Critically ill patients are at increased risk of fungal infections. Candida and Aspergillus are the most frequently isolated fungi in the intensive care unit (ICU). In fact, Candida is the third most prevalent microorganism in patients with infections the ICU [1]. It is responsible for $12 \%$ of ICU-acquired bloodstream infections [2]. Nosocomial Candida bloodstream infection (CBSI) is associated with increased morbidity and mortality as well as a prolonged hospital length of stay compared with other bloodstream infections [3]. Crude mortalities of $40-60 \%$

\footnotetext{
* Correspondence: yiorgos.alexandros.cavayas@umontreal.ca

${ }^{1}$ Département de Soins Critiques, Hôpital Sacré-Coeur de Montréal, 5400

Boul Gouin Ouest, Montreal, QC H4J 1C5, Canada

Full list of author information is available at the end of the article
}

and attributable mortalities of $5-71 \%$ have been reported in patients with Candida infection in the ICU [4-6]. Invasive aspergillosis (IA) has typically been described in patients with hematological malignancies and profound immunosuppression, but recent evidence has suggested that it may be present in a significant proportion of critically ill patients without these conventional risk factors [7]. Researchers in previous studies have reported a $1-2 \%$ prevalence of Aspergillus [8-11] in the respiratory tract of mechanically ventilated patients and up to $8 \%$ in those with acute respiratory distress syndrome (ARDS) [12]. In an autopsy series of patients who died of ARDS, a prevalence of $12.5 \%$ was described [13]. Even in the absence of hematological malignancy, isolation of Aspergillus in 
critically ill patients has been associated with mortality as high as $86 \%$ [14].

In the last decade, use of extracorporeal membrane oxygenation (ECMO) has significantly increased in the adult population [15]. It is now routinely used to support critically ill patients with severe respiratory or cardiac failure in whom conventional therapies have failed. The severity of their illness and blood circuit interactions are thought to impair their immune system. They have catheters, cannulas, and oxygenators that can become colonized with microorganisms. They are often treated with broad-spectrum antibacterial agents. These factors are thought to predispose them to fungal colonization and infection. Recently reported data suggest that up to $15 \%$ of all bloodstream infections while on ECMO are of fungal origin [16]. A 6.4\% prevalence of CBSI in an Australian center has been reported [17]. In another study, yeast DNA was retrieved in 7\% of oxygenators after weaning [18].

All of these studies were performed in single centers. Fungal infection rates can vary greatly between units, depending on the type of patients treated and differences in local antibiotic and antifungal prescription practices. Robust multicenter data are lacking. Therefore, the objective of the present study was to establish the prevalence, risk factors, and prognosis of fungal infection and colonization in an adult ECMO population composed of a large international cohort of patients.

\section{Methods}

\section{Study design}

We performed a retrospective cohort study using data from the Extracorporeal Life Support Organization (ELSO) registry. The registry compiles data on ECMO use in more than 300 international centers after approval by local institutional review boards. For each ECMO run, participating centers complete a standardized data sheet containing patient demographics, diagnosis and procedure information, ECMO technique, physiological and microbiological data, complications, and outcomes (see Additional file 1: Appendix 1). After approval by the ELSO Registry Committee, limited de-identified datasets are released to participating centers for research purposes without the need for further approval from individual centers. This study was conducted in accordance with the amended Declaration of Helsinki.

\section{Population}

We included all consecutive adult patients with fungal infection or colonization from January 2006 to September 2016 on any mode of extracorporeal life support. These patients were identified as those having either a diagnosis of fungal infection according to the International
Classification of Diseases, Ninth Revision, or any positive fungal culture (see Additional file 1: Appendix 2).

\section{Statistical analysis}

Analyses were performed using IBM SPSS Statistics for Mac software version 24.0 (IBM, Armonk, NY, USA). Categorical variables were summarized using frequencies and percentages, and continuous variables were summarized using mean and SD. Pearson's chi-square test was used to test for univariate associations of categorical variables, and the Mann-Whitney $U$ test was used for continuous variables. Missing variables were excluded from analyses.

To determine factors independently associated with $A s$ pergillus involvement (colonization or infection) and Candida bloodstream infection, we performed a multivariable logistic regression using a stepwise backward selection model with a $p$ value less than 0.20 for inclusion and greater than 0.15 for exclusion. In order to determine if $A s-$ pergillus involvement and CBSI were independently associated with a lower survival rate, we also used a multivariable logistic regression with a stepwise backward selection model with the same conditions for inclusion and exclusion. All of the models were validated by bootstrapping with 1000 samples. The variables entered into the models can be found in Additional file 1: Appendixes 5-7.

\section{Results}

There were 19,697 adult patients in ELSO registry during the study period. Of these, 2129 had either a diagnosis of fungal infection or a positive fungal culture, resulting in a prevalence of $10.8 \%$ (95\% CI $10.4-11.2 \%$ ) of infection and colonization. Their mean age was 48 . 5 years. The majority $(67.3 \%)$ of patients received ECMO for respiratory support, and a majority $(57.8 \%)$ were on venovenous ECMO (Table 1). The overall survival of patients with fungus did not significantly differ from the survival of those without $(49.7 \%$ vs $48.7 \% ; p=0.559)$. However, two main subgroups emerged as having a different survival in univariate analyses: patients with an aspergillosis diagnosis or any Aspergillus-positive culture (35.7\%; $p<0.001)$ and those with Candida in blood culture (35.9\%; $p<0.001)$ (Fig. 1). There were 272 patients with Aspergillus involvement in the registry, which resulted in a prevalence of $1.4 \%$ (95\% CI $1.2-1.5 \%)$, and 245 patients with CBSI, which resulted in a prevalence of $1.4 \% 1.2 \%$ (95\% CI 1.1-1.4\%) (Table 2). Positive Candida cultures in sites other than blood did not affect survival, no matter how many sites were affected (see Additional file 1: Appendix 4).

In multivariable analysis, male sex, hematological malignancy, influenza, solid organ transplant, and ECMO for respiratory support were associated with an increased risk of Aspergillus involvement. Increased weight, nonviral pneumonia, aspiration pneumonitis, ARDS, and 
Table 1 Characteristics of patients with fungal colonization or infection

\begin{tabular}{lll}
\hline Variable & Summary $(N=2129)$ \\
\hline Age, years, mean \pm SD & $48.5 \pm 15.7$ & \\
Sex, male, $n$ (\%) & 1264 & $(59.7 \%)$ \\
Weight, kg, mean \pm SD & $85.1 \pm 26.7$ & \\
Support type, $n$ (\%) & & \\
$\quad$ Respiratory & 1433 & $(67.3 \%)$ \\
$\quad$ Cardiac & 563 & $(26.4 \%)$ \\
$\quad$ ECPR & 133 & $(6.2 \%)$ \\
ECMO configuration, $n$ (\%) & & \\
$\quad$ VA only & 742 & $(34.8 \%)$ \\
$\quad$ W only & 1231 & $(57.8 \%)$ \\
$\quad$ Hybrid or conversion & 134 & $(6.3 \%)$ \\
Sepsis, $n$ (\%) & 505 & $(23.7 \%)$ \\
Pneumonia, $n$ (\%) & 464 & $(14.1 \%)$ \\
Influenza, $n$ (\%) & 301 & $(14.0 \%)$ \\
Acute respiratory distress syndrome, $n$ (\%) & 297 & $(33.4 \%)$ \\
Other acute respiratory failure, $n$ (\%) & 711 & $(26.0 \%)$ \\
Heart failure, $n$ (\%) & 554 & \\
\hline
\end{tabular}

Abbreviations: ECPR Extracorporeal cardiopulmonary resuscitation, $V A$ Venoarterial, $W V$ Venovenous acute respiratory failure were associated with a decreased risk of Aspergillus involvement (Table 3). With bootstrapping, the associations with hematological malignancies and ARDS were not statistically significant (see Additional file 1: Appendix 5). Although steroid administration was positively associated with Aspergillus involvement in univariate analysis (OR 1.50, 95\% CI 1. $07-2.10, p=0.017$ ), this association did not reach statistical significance in the multivariable backward selection model. There was no significant association between $A s$ pergillus involvement and chronic pulmonary condition, tobacco use, tuberculosis, or human immunodeficiency virus (HIV).

Advanced age, increased weight, sepsis, and renal replacement therapy (RRT) were independent risk factors for CBSI. Although increased weight, nonviral pneumonia, intra-aortic balloon pump, and cardiopulmonary bypass were associated with the risk of CBSI in the backward selection model, the association could not be validated by bootstrapping $(p>0.05)$ (Table 4; see also Additional file 1: Appendix 6]. Diabetes, steroids, femoral cannulation, laparotomy, and pancreatitis were not significantly associated with CBSI. The registry does not collect information on antibiotic or parenteral nutrition use.

As hypothesized, Aspergillus involvement (OR 0.40, $p$ $<0.001$ ) and CBSI (OR 0.47, $p<0.001$ ) were both independently associated with decreased survival. The other factors associated with worse survival in multivariable analysis were advanced age, hematological malignancy, acute kidney injury (AKI), HIV, ARDS, sepsis, nitric

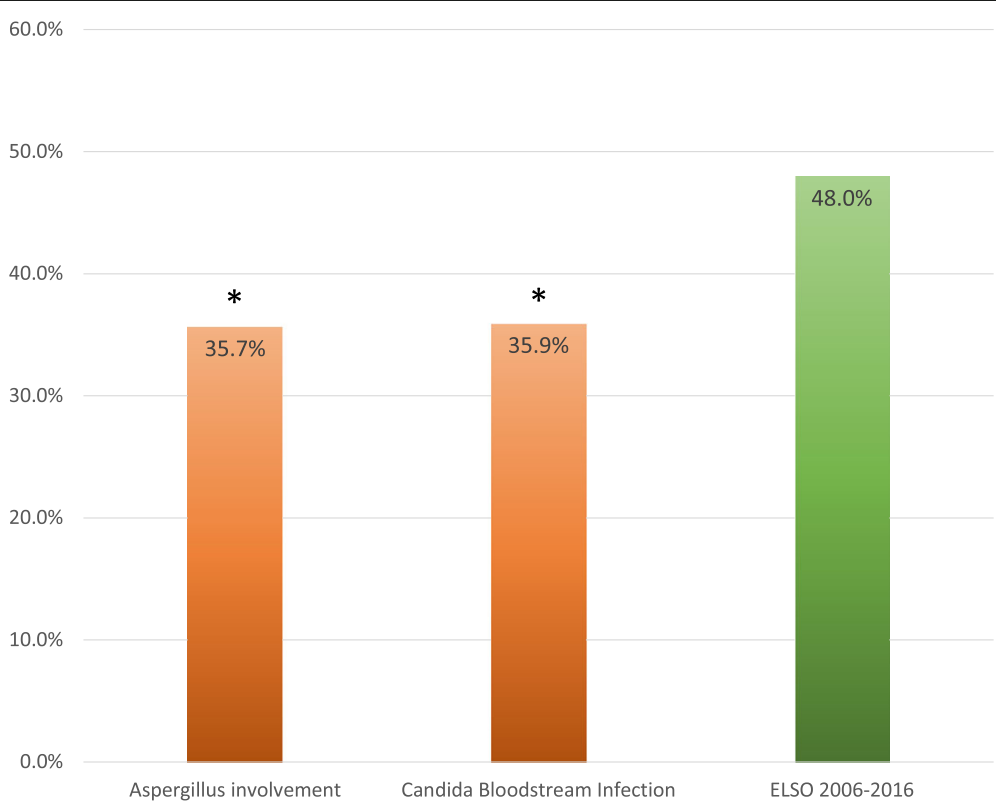

Fig. 1 Survival of patients with Aspergillus involvement and Candida bloodstream infection compared with the overall survival in the Extracorporeal Life Support Organization (ELSO) registry during study the period. *Statistically significant difference compared with overall survival in the ELSO registry during the study period 
Table 2 Prevalence and outcome of colonization and fungal infections

\begin{tabular}{|c|c|c|c|c|c|c|c|c|}
\hline \multirow[t]{2}{*}{ Fungus/diagnosis } & \multirow{2}{*}{$\begin{array}{l}\text { Total } \\
\text { No. }\end{array}$} & \multirow{2}{*}{$\begin{array}{l}\text { Prevalence } \\
\%\end{array}$} & \multirow{2}{*}{$\begin{array}{l}\text { Survival } \\
\%\end{array}$} & \multicolumn{2}{|c|}{ Survivors } & \multicolumn{2}{|c|}{ Nonsurvivors } & \multirow[b]{2}{*}{ Chi-square $p$ value } \\
\hline & & & & No. & $\%$ & No. & $\%$ & \\
\hline Diagnosis of aspergillosis & 69 & $0.4 \%$ & $28 \%$ & 19 & $1.8 \%$ & 50 & $4.7 \%$ & 0.003 \\
\hline Culture of Aspergillus without diagnosis & 203 & $1.0 \%$ & $38 \%$ & 78 & $7.4 \%$ & 125 & $12 \%$ & 0.009 \\
\hline Aspergillus (all) & 272 & $1.4 \%$ & $36 \%$ & 97 & $9.2 \%$ & 175 & $16 \%$ & $<0.001$ \\
\hline Diagnosis of systemic candidiasis & 27 & $0.1 \%$ & $44 \%$ & 12 & $1.1 \%$ & 15 & $1.4 \%$ & 0.959 \\
\hline Culture of Candida in blood & 245 & $1.2 \%$ & $36 \%$ & 88 & $8.3 \%$ & 157 & $15 \%$ & $<0.001$ \\
\hline Culture of Candida in respiratory tract & 1254 & $6.4 \%$ & $55 \%$ & 688 & $65 \%$ & 566 & $53 \%$ & $<0.001$ \\
\hline Culture of Candida in urine & 295 & $1.5 \%$ & $53 \%$ & 155 & $15 \%$ & 140 & $13 \%$ & 0.783 \\
\hline Candida (all) & 1907 & $9.7 \%$ & $51 \%$ & 977 & $92 \%$ & 930 & $87 \%$ & 0.001 \\
\hline Diagnosis of blastomycosis & 17 & $0.1 \%$ & $53 \%$ & 9 & $0.8 \%$ & 8 & $0.7 \%$ & 0.995 \\
\hline Diagnosis of other fungal infection & 33 & $0.2 \%$ & $42 \%$ & 14 & $1.3 \%$ & 19 & $1.8 \%$ & 0.869 \\
\hline Total & 2129 & $11 \%$ & $50 \%$ & 1059 & - & 1070 & - & - \\
\hline
\end{tabular}

oxide, ECMO for cardiac support, and extracorporeal cardiopulmonary resuscitation. However, increased weight, aspiration pneumonitis, influenza, solid organ transplant, nonviral pneumonia, and neuromuscular blockers were independently associated with increased survival in our cohort (Table 5). The associations with AKI, sepsis, and solid organ transplant were not statistically significant with bootstrapping (see also Additional file 1: Appendix 7).

\section{Discussion}

We found a $10.8 \%$ prevalence of fungal infection or colonization in the ELSO registry, including $1.3 \%$ of patients with Aspergillus involvement and $1.2 \%$ of patients with CBSI. These prevalence rates are lower than expected in a population of mechanically ventilated critically ill patients with indwelling catheters and an ICU length of stay generally exceeding 1 week. Aspergillus involvement was associated with classic factors that cause immunosuppression, such as hematological malignancy and solid organ transplant, as well as with influenza and ECMO for respiratory support. Not surprisingly, CBSI was associated with typical risk factors such as sepsis and RRT. The survival of patients with any Aspergillus involvement (37.5\%) was significantly lower than that of the rest of the ELSO population, even after adjusting for other predictive factors in multivariable analysis (OR 0 .

Table 3 Risk factors for Aspergillus colonization or infection

\begin{tabular}{llll}
\hline Variable & OR & $95 \% \mathrm{Cl}$ & $p$ Value \\
\hline Male sex & 1.55 & $1.16-2.08$ & 0.003 \\
Weight/10 kg & 0.87 & $0.82-0.93$ & $<0.001$ \\
Hematological malignancy & 2.18 & $0.99-4.78$ & 0.052 \\
Aspiration pneumonitis & 0.33 & $0.12-0.93$ & 0.036 \\
ARDS & 0.75 & $0.50-1.12$ & 0.154 \\
Acute respiratory failure NOS & 0.58 & $0.42-0.79$ & 0.001 \\
\hline
\end{tabular}

ARDS Acute respiratory distress syndrome, NOS Not otherwise specified
41). Similarly, CBSI was associated with poor survival (35.9\%) independently (OR 0.47).

The prevalence of fungus-positive samples in general ICU patients with a length of stay more than 7 days has been reported to be as high as $59.7 \%$ with systematic sampling, much higher than in our cohort [19]. However, cultures reported in the ELSO registry are performed at the discretion of clinicians rather than systematically. It is highly probable that fungi were simply undetected in a significant number of patients. This could have resulted in a significant underestimation of the overall prevalence of fungal colonization and infection as well as of the prevalence of specific infections. Moreover, information about antifungal agent administration was not available. A more liberal use of antifungal prophylaxis in this very sick population of patients could also have contributed to a lower rate of funguspositive samples.

The overall prevalence of any microbiological or histological evidence of Aspergillus in a medical ICU has been reported to be $6.9 \%$, and a combined prevalence of proven, probable, or possible IA has been reported to be $5.8 \%$ [14]. These data are more in line with the $7.2 \%$ prevalence found in an Australian ICU in a review of their ECMO cases between 2005 and 2011 [20]. There

Table 4 Risk factors for Candida bloodstream infection

\begin{tabular}{llll}
\hline Variable & OR & $95 \% \mathrm{Cl}$ & $p$ Value \\
\hline Age/10 years & 1.13 & $1.03-1.25$ & 0.011 \\
Weight/10 kg & 1.05 & $1.00-1.10$ & 0.061 \\
Sepsis & 1.60 & $1.15-2.23$ & 0.005 \\
Renal replacement therapy & 1.55 & $1.12-2.13$ & 0.007 \\
Nonviral pneumonia & 0.70 & $0.48-1.03$ & 0.068 \\
Intra-aortic balloon pump & 1.46 & $0.94-2.26$ & 0.091 \\
Cardiopulmonary bypass & 0.56 & $0.28-1.15$ & 0.112 \\
\hline
\end{tabular}


Table 5 Independent predictors of survival

\begin{tabular}{llll}
\hline Variable & OR & $95 \% \mathrm{Cl}$ & $p$ Value \\
\hline Age/10 years & 0.81 & $0.77-0.87$ & $<0.001$ \\
Weight/10 kg & 1.04 & $1.01-1.08$ & 0.018 \\
Hematological malignancy & 0.44 & $0.21-0.95$ & 0.035 \\
Acute kidney injury & 0.83 & $0.66-1.05$ & 0.116 \\
HIV infection & 0.09 & $0.02-0.42$ & 0.002 \\
Aspiration pneumonitis & 1.87 & $1.14-3.09$ & 0.014 \\
ARDS & 0.66 & $0.50-0.88$ & 0.004 \\
Influenza & 1.67 & $1.26-2.22$ & $<0.001$ \\
Sepsis & 0.80 & $0.63-1.02$ & 0.07 \\
Solid organ transplant & 1.37 & $0.96-1.96$ & 0.086 \\
Nonviral pneumonia & 1.33 & $1.05-1.68$ & 0.02 \\
Nitric oxide & 0.55 & $0.42-0.73$ & $<0.001$ \\
Neuromuscular blockers & 1.26 & $1.03-1.55$ & 0.027 \\
Cardiac support & 0.59 & $0.47-0.75$ & $<0.001$ \\
ECPR & 0.45 & $0.30-0.67$ & $<0.001$ \\
Candida bloodstream infection & 0.47 & $0.34-0.63$ & $<0.001$ \\
Aspergillus colonization or infection & 0.40 & $0.30-0.54$ & $<0.001$ \\
\hline Abbreviations: ARDS Acute respiratory distress syndrome, HIV Human &
\end{tabular}

immunodeficiency virus, ECPR Extracorporeal cardiopulmonary resuscitation

was a much lower prevalence of Aspergillus involvement in the ELSO registry. The first caveat in interpreting the rates of Aspergillus involvement is that microscopic examination and culture of respiratory tract specimens have a combined sensitivity of only $50 \%$ for IA [21]. Second, discriminating between colonization and infection is always challenging without histology. Biopsies are rarely performed in ECMO patients, because they are at high risk of bleeding owing to systemic anticoagulation, platelet and coagulation factor consumption, and increased fibrinolysis associated with the extracorporeal circuit. Nonneutropenic patients most often do not display the characteristic radiological signs of IA [22]. Furthermore, many patients had respiratory failure with already abnormal chest imaging results before developing IA. Newer tests such as galactomannan and PCR of serum or bronchoalveolar lavage specimens have been developed to improve the diagnostic yield for IA [23]. However, results of such tests, if performed, were not collected in the database. Blot and colleagues have described and validated an algorithm for use in diagnosing aspergillosis in critically ill individuals [24]. However, the ELSO registry did not collect all the variables needed to apply their definitions. In addition, whether Aspergillus isolated in the lower respiratory tract of critically ill patients can be viewed as a contaminant or colonizer remains a matter of debate $[25,26]$.

Meersseman and colleagues reported an overall survival of $20 \%$ in patients without hematological malignancy with evidence of Aspergillus involvement [14]. The higher survival that we found could be due to the fact that ECMO patients, although acutely very ill, are younger and have fewer comorbidities than the general ICU population. Patients with Aspergillus involvement in the ELSO registry had a mean age of 46 years, and only $8.5 \%$ of them had chronic pulmonary disease, as compared with a mean age of 61 years and $42 \%$ with chronic pulmonary disease in the Meersseman study.

The strong association between influenza and Aspergillus found in our cohort has been described before in case series and case-control studies. Coinfection rates ranging from $29 \%$ to $75 \%$ have been reported [27, 28]. Proposed mechanisms for this increased susceptibility to Aspergillus invasion include both local and systemic effects [29]. Indeed, influenza induces tracheitis and bronchitis and impairs normal ciliary function [30]. The virus also impairs phagocytosis and induces anti-inflammatory cytokine production leading to T-cell dysfunction and apoptosis [31-33].

In recent large multicentric cohort studies of unselected patients admitted to mixed ICUs, the prevalence of candidemia ranged from $0.33 \%$ to $0.69 \%$ [3, 34]. Although the rate we found was higher than the one reported in these large studies, in a cohort of patients with severe critical illness and a long ICU length of stay, we expected a much higher rate. A prevalence of $3.3 \%$ has been reported in patients with a length of stay in the ICU more than 7 days [19]. Moreover, a prevalence of 6 . $4 \%$ of CBSI was found in a retrospective review of ECMO cases in an Australian center [17]. In keeping with these findings, yeast DNA has been detected in 7\% of patients on oxygenators [18]. In theory, this should translate to positive fungal cultures at some point. The discrepancy could be explained by undersampling or a more liberal use of antifungal prophylaxis.

In contrast, CBSI survival was strikingly worse than previously described in the literature. León and colleagues found an overall survival of $43.4 \%$ in critically ill patients with invasive Candida infection hospitalized for more than 7 days [19]. Others have even found a survival of $57-60 \%$ in general ICU cohorts [3,34]. Patients on ECMO could simply have a higher illness severity than these cohorts. It is also possible that the presence of foreign material makes it more difficult to eradicate Candida, as seen with prosthetic valve endocarditis.

Multiple hospitals across the globe in very different settings participate in the ELSO registry. These centers collect data on all their ECMO cases. This confers strong external validity to our study. However, the internal validity of the study is affected by multiple factors partly inherent to its retrospective nature. For instance, microbiological sampling was nonsystematic. This may have caused underestimation of the prevalence of fungal 
infections. This also weakened the analysis of risk factors by making them highly susceptible to observer bias. Indeed, fungal cultures may have been performed more often in patients with presenting factors that were suspected by clinicians to increase the risk of fungal infection. Moreover, limited data were available on potential confounding factors such as antifungal therapy and prophylaxis. This may have decreased certain associations with risk factors if antifungal therapy was prescribed more often in those patients. Such limitations could be avoided or minimized in a prospective study.

\section{Conclusions}

Patients on ECMO do not seem to develop fungal colonization or infection more frequently than other critically ill patients. Aspergillus involvement and CBSI were independently associated with decreased survival. CBSI mortality was higher than described in the general ICU population. Aspergillus involvement was associated with respiratory ECMO and influenza. Clinicians should maintain a high index of suspicion in this subgroup, and efforts should be made to establish an early diagnosis. It remains unclear, however, if treating the fungus would improve survival.

\section{Additional file}

Additional file 1: Appendix 1. ELSO Registry Case Report Form (document). Appendix 2. Patient selection (list). Appendix 3. Details of Aspergillus involvement (table). Appendix 4. Survival of patients according to number of cultures other than blood positive for Candida (table). Appendix 5. Multiple logistic regression for Aspergillus (table). Appendix 6. Multiple logistic regression for Candida bloodstream infection (table). Appendix 7. Multiple logistic regression for survival (table). Appendix 8. Case distribution by year (figure). (PDF $1090 \mathrm{~kb}$ )

\section{Abbreviations}

AKI: Acute kidney injury; ARDS: Acute respiratory distress syndrome; CBSI: Candida bloodstream infection; ECMO: Extracorporeal membrane oxygenation; ECPR: Extracorporeal cardiopulmonary resuscitation; ELSO: Extracorporeal Life Support Organization; HIV: Human immunodeficiency virus; IA: Invasive aspergillosis; ICU: Intensive care unit; RRT: Renal replacement therapy

\section{Acknowledgements}

The data were provided by Peter Rycus, executive administrator of the Extracorporeal Life Support Organization registry. We thank Peter Rycus, the ELSO registry, Gail Faulkner, and the rest of the Leicester ECMO team for their help and support

\section{Availability of data and materials \\ The data that support the findings of this study are available from the Extracorporeal Life Support Organization (ELSO), but restrictions apply to the availability of these data, which were used under license for the present study and so are not publicly available. Data are available from the authors, however, upon reasonable request and with permission of the ELSO.}

\section{Authors' contributions}

YAC and HY contributed to study design, data analysis and interpretation, and writing of the manuscript. RP contributed to the writing of the manuscript. All authors read and approved the final manuscript.

\section{Ethics approval and consent to participate}

After approval by the ELSO Registry Committee, limited de-identified datasets are released to participating centers for research purposes without the need for further approval from individual centers. No patient consent is required. This study was conducted in accordance with the amended Declaration of Helsinki.

\section{Competing interests}

The authors declare that they have no competing interests.

\section{Publisher's Note}

Springer Nature remains neutral with regard to jurisdictional claims in published maps and institutional affiliations.

\section{Author details}

'Département de Soins Critiques, Hôpital Sacré-Coeur de Montréal, 5400 Boul Gouin Ouest, Montreal, QC H4J 1C5, Canada. ${ }^{2}$ University Hospitals of Leicester, ECMO program, Glenfield Hospital, Groby Rd, Leicester LE3 9QP, UK.

Received: 17 January 2018 Accepted: 28 March 2018

Published online: 17 April 2018

\section{References}

1. Vincent JL, Rello J, Marshall J, et al. International study of the prevalence and outcomes of infection in intensive care units. JAMA. 2009;302:2323-9.

2. Hidron Al, Edwards JR, Patel J, et al. NHSN annual update: antimicrobialresistant pathogens associated with healthcare-associated infections: annual summary of data reported to the National Healthcare Safety Network at the Centers for Disease Control and Prevention, 2006-2007. Infect Control Hosp Epidemiol. 2008;29:996-1011. A published erratum appears in Infect Control Hosp Epidemiol. 2009:30:107

3. Kett DH, Azoulay E, Echeverria PM, et al. Candida bloodstream infections in intensive care units: analysis of the extended prevalence of infection in intensive care unit study. Crit Care Med. 2011;39:665-70.

4. Méan M, Marchetti O, Calandra T. Bench-to-bedside review: Candida infections in the intensive care unit. Crit Care. 2008;12:204.

5. Guery BP, Arendrup MC, Auzinger $G$, et al. Management of invasive candidiasis and candidemia in adult non-neutropenic intensive care unit patients: part II. Treatment. Intensive Care Med. 2009:35:206-14.

6. Delaloye J, Calandra T. Invasive candidiasis as a cause of sepsis in the critically ill patient. Virulence. 2014;5:161-9.

7. Koulenti D, Garnacho-Montero J, Blot S. Approach to invasive pulmonary aspergillosis in critically ill patients. Curr Opin Infect Dis. 2014;27:174-83.

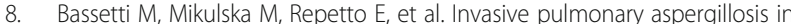
intensive care units: is it a real problem? J Hosp Infect. 2010;74:186-7.

9. Garnacho-Montero J, Amaya-Villar R, Ortiz-Leyba C, et al. Isolation of Aspergillus spp. from the respiratory tract in critically ill patients: risk factors, clinical presentation and outcome. Crit Care. 2005;9:R191-9.

10. Vandewoude $\mathrm{K}$, Blot $\mathrm{S}$, Benoit $\mathrm{D}$, et al. Invasive aspergillosis in critically ill patients: analysis of risk factors for acquisition and mortality. Acta Clin Belg. 2004;59:251-7.

11. Vandewoude KH, Blot SI, Depuydt P, et al. Clinical relevance of Aspergillus isolation from respiratory tract samples in critically ill patients. Crit Care. 2006;10:R31.

12. Contou D, Dorison M, Rosman J, et al. Aspergillus-positive lower respiratory tract samples in patients with the acute respiratory distress syndrome: a 10year retrospective study. Ann Intensive Care. 2016;6:52.

13. de Hemptinne Q, Remmelink M, Brimioulle S, et al. ARDS: a clinicopathological confrontation. Chest. 2009:135:944-9.

14. Meersseman W, Vandecasteele SJ, Wilmer A, et al. Invasive aspergillosis in critically ill patients without malignancy. Am J Respir Crit Care Med. 2004 170:621-5.

15. Karagiannidis C, Brodie D, Strassmann S, et al. Extracorporeal membrane oxygenation: evolving epidemiology and mortality. Intensive Care Med. 2016:42:889-96.

16. Kim DW, Yeo HJ, Yoon SH, et al. Impact of bloodstream infections on catheter colonization during extracorporeal membrane oxygenation. J Artif Organs. 2015:19:128-33.

17. Aubron C, Cheng AC, Pilcher D, et al. Infections acquired by adults who receive extracorporeal membrane oxygenation risk factors and outcome. Infect Control Hosp Epidemiol. 2015;34:24-30 
18. Kuehn C, Orszag P, Burgwitz K, et al. Microbial adhesion on membrane oxygenators in patients requiring extracorporeal life support detected by a universal rDNA PCR test. ASAIO J. 2013;59:368-73.

19. León C, Álvarez-Lerma F, Ruiz-Santana S, et al. Fungal colonization and/or infection in non-neutropenic critically ill patients: results of the EPCAN observational study. Eur J Clin Microbiol Infect Dis. 2009;28:233-42.

20. Aubron C, Pilcher D, Leong T, et al. Aspergillus sp. isolated in critically ill patients with extracorporeal membrane oxygenation support. Scand I Infect Dis. 2013;45:715-21.

21. Hope WW, Walsh TJ, Denning DW. Laboratory diagnosis of invasive aspergillosis. Lancet Infect Dis. 2005;5:609-22.

22. Meersseman W, Lagrou K, Maertens J, et al. Invasive aspergillosis in the intensive care unit. Clin Infect Dis. 2007:45:205-16.

23. Meersseman W, Lagrou K, Maertens J, et al. Galactomannan in bronchoalveolar lavage fluid. Am J Respir Crit Care Med. 2008;177:27-34.

24. Blot SI, Taccone FS, Van den Abeele AM, et al. A clinical algorithm to diagnose invasive pulmonary aspergillosis in critically ill patients. Am J Respir Crit Care Med. 2012;186:56-64.

25. Alshabani K, Haq A, Miyakawa R, et al. Invasive pulmonary aspergillosis in patients with influenza infection: report of two cases and systematic review of the literature. Expert Rev Respir Med. 2014;9:89-96.

26. Khasawneh F, Mohamad T, Moughrabieh MK, et al. Isolation of Aspergillus in critically ill patients: a potential marker of poor outcome. J Crit Care. 2006; 21:322-7.

27. Guervilly C, Roch A, Ranque S, et al. A strategy based on galactomannan antigen detection and $\mathrm{PCR}$ for invasive pulmonary aspergillosis following influenza A (H1N1) pneumonia. J Infect. 2012;65:470-3.

28. Crum-Cianflone NF. Invasive aspergillosis associated with severe influenza infections. Open Forum Infect Dis. 2016;3:171-8.

29. Lewis M, Kallenbach J, Ruff $P$, et al. Invasive pulmonary aspergillosis complicating influenza A pneumonia in a previously healthy patient. Chest. 1985;87:691-3.

30. Gill JR, Sheng ZM, Ely SF, et al. Pulmonary pathologic findings of fatal 2009 pandemic influenza A/H1N1 viral infections. Arch Pathol Lab Med. 2010;134:235-43.

31. Adalja AA, Sappington PL, Harris SP, et al. Isolation of Aspergillus in three 2009 H1N1 influenza patients. Influenza Other Respir Viruses. 2011:5:225-9.

32. Hinshaw VS, Olsen CW, Dybdahl-Sissoko N, et al. Apoptosis: a mechanism of cell killing by influenza A and B viruses. J Virol. 1994;68:3667-73.

33. Xie D, Bai H, Liu L, et al. Apoptosis of lymphocytes and monocytes infected with influenza virus might be the mechanism of combating virus and causing secondary infection by influenza. Int Immunol. 2009;21:1251-62.

34. Shahin J, Allen EJ, Patel $\mathrm{K}$, et al. Predicting invasive fungal disease due to Candida species in non-neutropenic, critically ill, adult patients in United Kingdom critical care units. BMC Infect Dis. 2016;16:480

\section{Submit your next manuscript to BioMed Central and we will help you at every step:}

- We accept pre-submission inquiries

- Our selector tool helps you to find the most relevant journal

- We provide round the clock customer support

- Convenient online submission

- Thorough peer review

- Inclusion in PubMed and all major indexing services

- Maximum visibility for your research

Submit your manuscript at www.biomedcentral.com/submit

) Biomed Central 